\title{
SINAIS DE URETEROLITÍASE NA TOMOGRAFIA COMPUTADORIZADA HELICOIDAL SEM CONTRASTE: ENSAIO ICONOGRÁFICO E REVISÃO DA LITERATURA*
}

\author{
Renata La Rocca Vieira ${ }^{1}$, Salomão Faintuch ${ }^{1}$, Suzan Menasce Goldman ${ }^{2}$, Sérgio Aron Ajzen ${ }^{3}$, \\ Jacob Szejnfeld ${ }^{4}$
}

\begin{abstract}
Resumo 0 principal sinal diagnóstico de ureterolitíase na tomografia computadorizada é a visualização direta do cálculo no interior do ureter. Todavia, a sua caracterização pode ser prejudicada devido a suas pequenas dimensões, variação da respiração entre a aquisição dos cortes tomográficos, escassez de gordura retroperitoneal ou eliminação recente do cálculo. Neste contexto, foram descritos diversos sinais secundários de obstrução ureteral, observados na tomografia computadorizada, que podem auxiliar no diagnóstico de casos duvidosos, além de quantificar o grau de obstrução urinária.
\end{abstract}

Unitermos: Tomografia computadorizada; Cólica renal; Cálculo.

Abstract Ureterolithiasis signs on unenhanced helical computed tomography: iconographic essay and literature review. The most important diagnostic sign of ureterolithiasis is the direct visualization of the stone within the ureter. However, in some patients with ureterolithiasis stone visualization may be impaired due to stone small size or low attenuation, respiratory artifacts between data acquisitions, low amount of retroperitoneal fat or recent elimination of the stone. In this context, we describe many secondary computed tomography signs of ureteral obstruction that may useful in the diagnosis of inconclusive cases, and also quantify the degree of urinary obstruction.

Key words: Computed tomography; Acute flank pain; Ureteral stone.

\section{INTRODUÇÃO}

A tomografia computadorizada (TC) helicoidal não contrastada tem sido o exame de escolha para a avaliação de pacientes com suspeita de cólica renal, desde a sua introdução em 1995 por Smith et al. ${ }^{(\mathbf{1})}$. É um método rápido, não invasivo e isento dos riscos relacionados ao uso endovenoso do meio de contraste iodado. Além disso, possui alta sensibilidade $(96 \%)$ e especificidade $(100 \%)$ para o diagnóstico de ureterolitíase e permite a elucidação de outras causas de dor no flanco, como apendicite, pielonefrite ou colecistite ${ }^{(2)}$.

O principal sinal diagnóstico de ureterolitíase na TC é a visualização direta do

* Trabalho realizado no Departamento de Diagnóstico por Imagem da Universidade Federal de São Paulo/Escola Paulista de Medicina (Unifesp/EPM), São Paulo, SP.

1. Médicos Residentes do Departamento de Diagnóstico por Imagem da Unifesp/EPM.

2. Médica Doutora do Setor de Abdome do Departamento de Diagnóstico por Imagem da Unifesp/EPM.

3. Professor Adjunto Livre-Docente do Departamento de Diagnóstico por Imagem da Unifesp/EPM.

4. Professor Livre-Docente, Chefe do Departamento de Diagnóstico por Imagem da Unifesp/EPM.

Endereço para correspondência: Dra. Renata La Rocca Vieira. Rua Doutor Diogo de Faria, 650/33, Vila Clementino. São Paulo, SP, 04037-002. E-mail: relarocca@uol.com.br

Recebido para publicação em 19/3/2003. Aceito, após revisão, em 22/12/2003. cálculo no interior do ureter. Todavia, a sua caracterização pode ser prejudicada devido a suas pequenas dimensões, variação da respiração entre a aquisição dos cortes tomográficos, escassez de gordura retroperitoneal ou eliminação recente do cálculo ${ }^{(3)}$. Devemos salientar que cálculos secundários à deposição de cristais de indinavir (inibidor de protease) são radiotransparentes na tomografia e de difícil diagnóstico ${ }^{(4)}$. A presença de calcificações pélvicas (flebólitos) pode ser causa de confusão diagnóstica $^{(5)}$.

Neste contexto, foram descritos diversos sinais secundários de litíase ureteral, observados na TC, que podem auxiliar no diagnóstico de casos duvidosos, além de quantificar o grau de obstrução urinária ${ }^{(3)}$.

\section{SINAIS SECUNDÁRIOS DE LITÍASE URETERAL}

Dilatação ureteral unilateral: É caracterizada pelo diâmetro ureteral maior que $2 \mathrm{~mm}^{(\mathbf{6})}$, sendo o sinal secundário com maior sensibilidade (90\%). Apresenta também alta especificidade $(93 \%)^{(\mathbf{3})}$. O seu surgimento é precoce, estando presente em $84 \%$ dos pacientes com cólica renal com duração inferior a duas horas ${ }^{(6)}$.

Opacificação da gordura perirrenal: É um achado comum, principalmente em

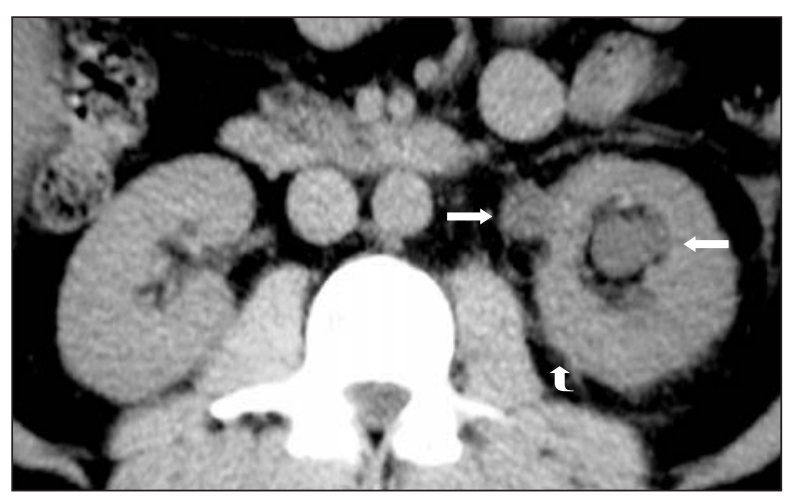

Figura 1. Tomografia computadorizada helicoidal do abdome sem contraste evidencia dilatação calicopieloureteral esquerda (setas), com heterogeneidade da gordura perirrenal homolateral (seta curva). 
idosos, secundário a aumento da pressão linfática regional. Pode estar presente em apenas um dos pólos renais. Apresenta alta especificidade $(93 \%)$, porém baixa sensibilidade $(83 \%)^{(3)}$. Pode ser graduado em leve, moderado e grave. Sua prevalência não é alterada de maneira significativa com o aumento do tempo de dor. Segundo um trabalho recente, suas freqüências nas três e cinco primeiras horas foram de $79 \%$ e $72 \%$, respectivamente ${ }^{(6)}$.

Dilatação do sistema coletor: A dilatação do sistema coletor é mais bem iden- tificada nos pólos renais, ao passo que a dilatação da porção central do sistema coletor deve ser vista com cautela, uma vez que também pode corresponder à pelve extrarenal. Apresenta alta especificidade (94\%) e baixa sensibilidade $(83 \%)^{(3)}$. Sua frequiência nas duas e oito primeiras horas de dor é de $68 \%$ e $89 \%$, o que constitui um aumento significante ${ }^{(6)}$. Sua quantificação é realizada de forma visual e subjetiva.

Nefromegalia: Apresenta sensibilidade (71\%) e especificidade ( $89 \%$ ) relativamente baixas. A sua frequiência de apresenta- ção, segundo Varanelli et al., não é influenciada pela duração da dor ${ }^{(\mathbf{6})}$.

Líquido perirrenal: A presença de moderada ou grande quantidade de líquido perirrenal está diretamente relacionada à duração da dor, com uma frequiência de $22 \%$ nas oito primeiras horas de cólica ${ }^{(6)}$.

Ausência unilateral da pirâmide renal: É um achado descrito recentemente. Consiste na perda da hiperatenuação espontânea das pirâmides renais do lado obstruído. Suas sensibilidade e especificidade ainda não foram caracterizadas ${ }^{(4,7)}$.

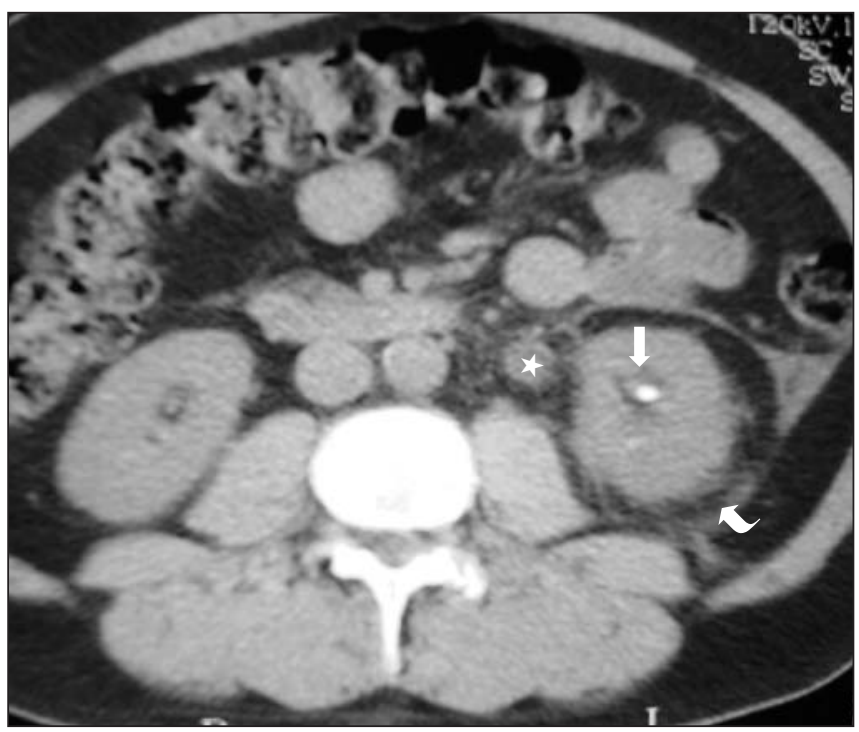

Figura 2. Tomografia helicoidal do abdome sem contraste evidencia cálculo no grupamento calicial médio esquerdo (seta), associado a heterogeneidade da gordura perirrenal (seta curva) e dilatação ureteral (estrela).

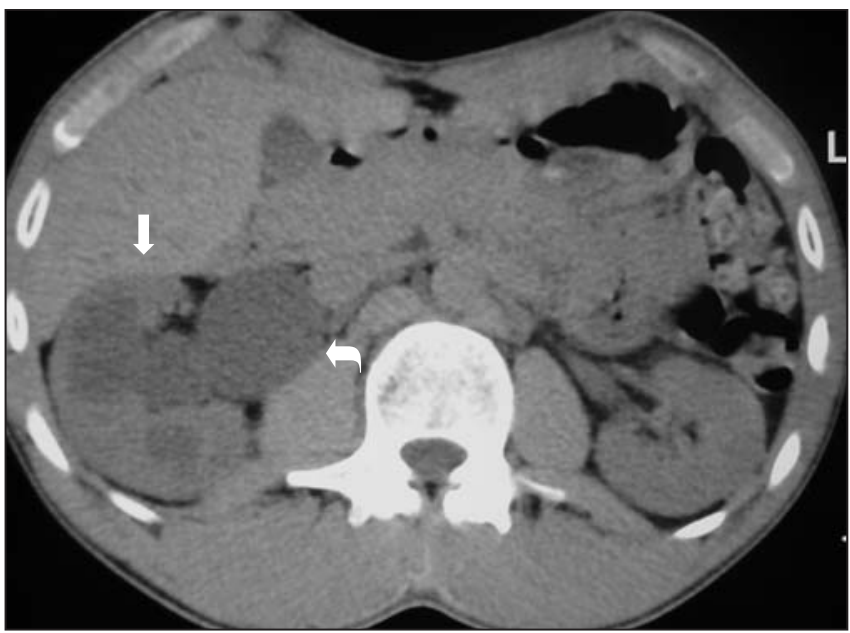

A

Figura 4. Nefromegalia. Tomografia computadorizada helicoidal do abdome sem contraste evidencia rins aumentados em tamanho (setas). Além disso, nota-se dilatação calicopieloureteral (setas curvas).

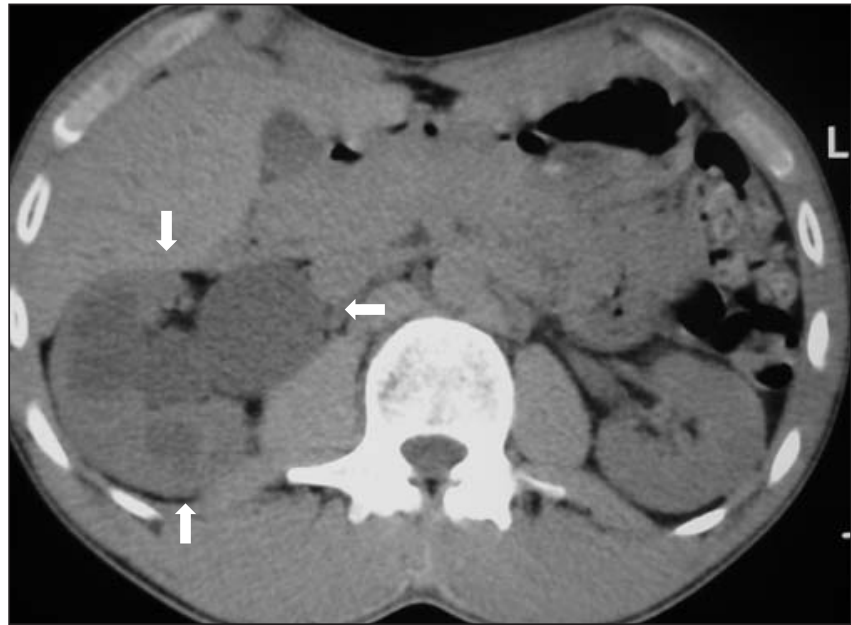

Figura 3. Tomografia helicoidal sem contraste do abdome evidencia nefromegalia e dilatação calicopieloureteral direita (setas).

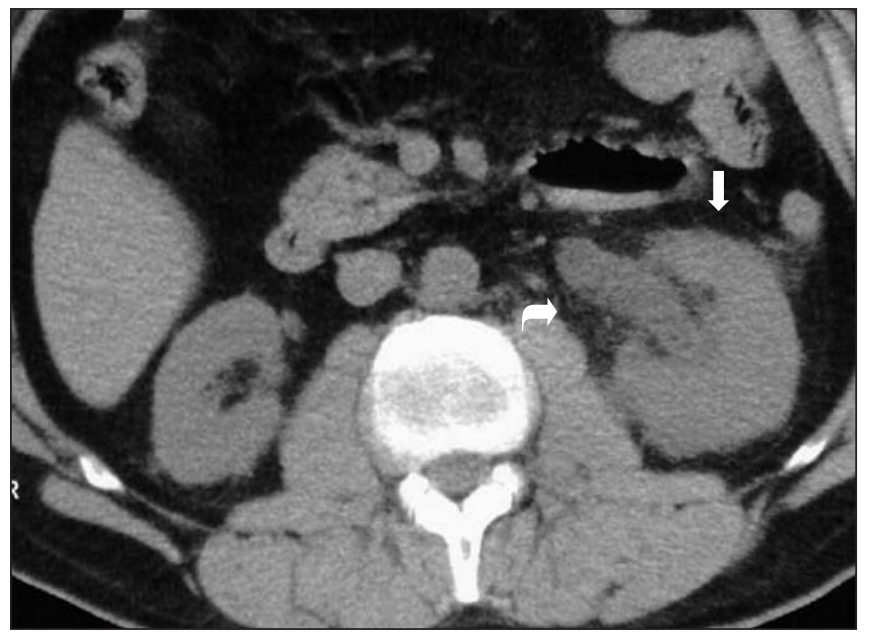

B 
Figura 5. Sinal da pirâmide hiperdensa. Tomografia computadorizada helicoidal sem contras te do abdome evidencia hiperdensidade na pirâmide rena direita (seta) e ausência da mesma no rim contralateral, indicando processo obstrutivo no rim esquerdo.

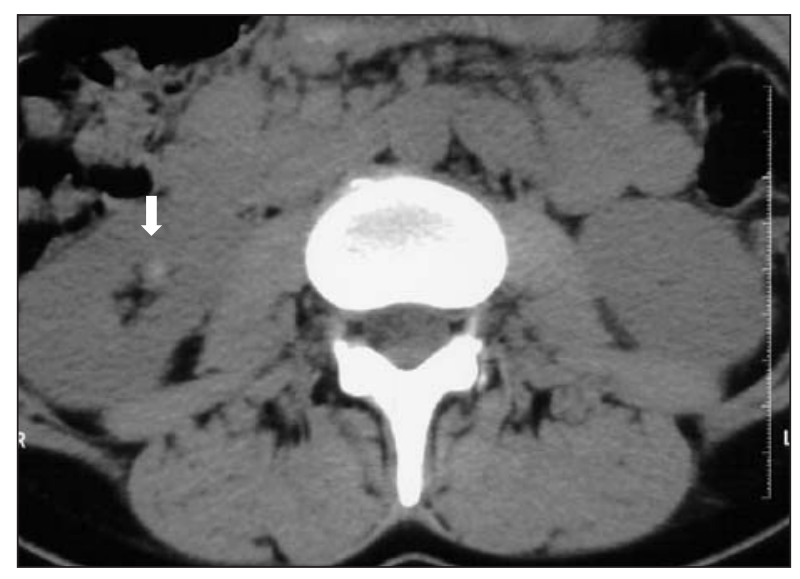

\section{SINAIS DIRETOS DE LITÍASE URETERAL}

Após a pesquisa de sinais secundários obstrutivos, segue-se a investigação de calcificações. Para a caracterização destas, recomenda-se acompanhar todo o trajeto do ureter, da pelve renal até a bexiga, para a identificação da calcificação no interior da luz ureteral. Porém, esta tarefa pode não ser simples, devido à escassez da gordura retroperitoneal em alguns pacientes, principalmente na região pélvica.

Sinal do halo ureteral: $O$ sinal do halo, ou do anel, ureteral representa o edema que circunda o cálculo, não sendo encontrado nas calcificações vasculares. Sua sensibilidade é de 77\%, segundo Katz et $a{ }^{(8)}$. É importante salientar que a presença ou ausência dos sinais obstrutivos secundários é de grande importância na di- ferenciação entre flebólito e cálculo, uma vez que é rara a ausência de sinais obstrutivos na presença do cálculo ureteral ${ }^{(5)}$.

\section{SINAIS UTILIZADOS PARA DIFERENCIAÇÃO ENTRE LITÍASE URETERAL E CALCIFICAÇÕES PÉLVICAS (FLEBÓLITOS)}

Sinal da cauda de cometa: $O$ sinal da cauda de cometa compreende uma estrutura linear ou curvilínea, de densidade de partes moles, que se estende a partir da calcificação vascular. Sua ausência não descarta a possibilidade de flebólito ${ }^{(5,9)}$.

Outra possibilidade de se caracterizar a calcificação pélvica é acompanhar todo o trajeto ureteral, assim como identificar o sinal do halo, já descrito. As calcificações pélvicas na altura ou inferiormente ao colo uterino são flebólitos, pois as junções ure- terovesicais situam-se acima desta estrutura. As calcificações vasculares podem ser radiotransparentes no seu centro e sempre acompanham o trajeto do vaso ${ }^{(9)}$.

\section{CONSIDERAÇÕES TÉCNICAS}

Utiliza-se técnica helicoidal, sem contraste oral ou endovenoso, colimação de 3 ou 5 mm e "pitch" de 1 . As imagens são obtidas desde os pólos superiores dos rins até a sínfise púbica. Reconstruções multiplanares podem ser necessárias para melhor ilustrar o cálculo e suas relações anatômicas, por isso a não administração do contraste por via oral ${ }^{(3,4,6)}$.

$\mathrm{Na}$ presença de cálculo no interior da bexiga deve-se realizar cortes tomográficos com o paciente em decúbito ventral, o que permite caracterizar se o cálculo encontrase fixo à junção ureterovesical ou não, com base na sua mobilidade ${ }^{(4)}$.

Todos os cálculos urinários, inclusive os de ácido úrico, são radiopacos à TC, exceto aqueles secundários à deposição de cristais de indinavir ${ }^{(4)}$. Com o advento dos anti-retrovirais, tem-se observado a presença de cálculos radiotransparentes na TC. Nestes pacientes, a identificação de sinais secundários assume importância maior para o diagnóstico.

A TC helicoidal é o melhor método no diagnóstico de litíase ureteral, e o conhecimento dos sinais de ureterolitíase na TC tem grande utilidade no diagnóstico de casos duvidosos. Dentre estas situações, merecem menção os cálculos radiotranspa-

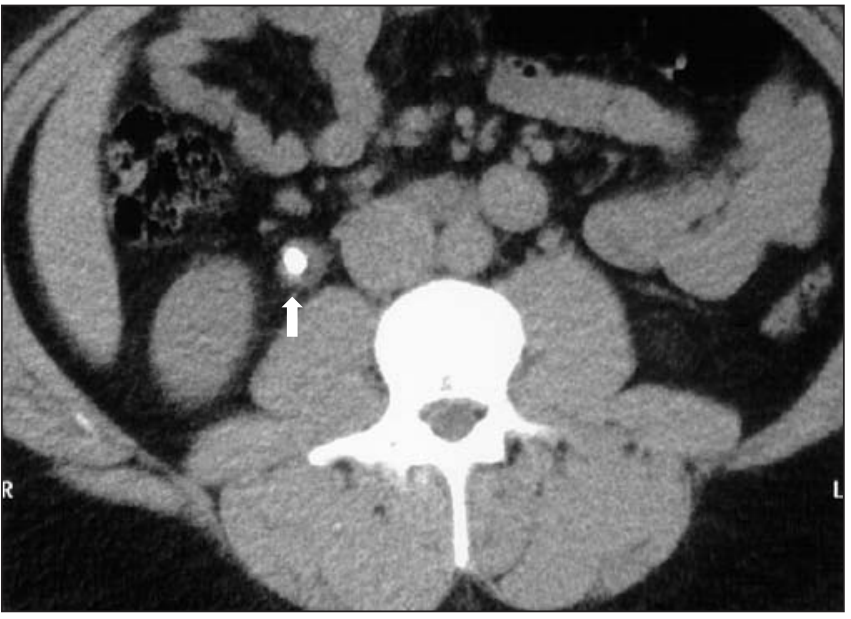

A

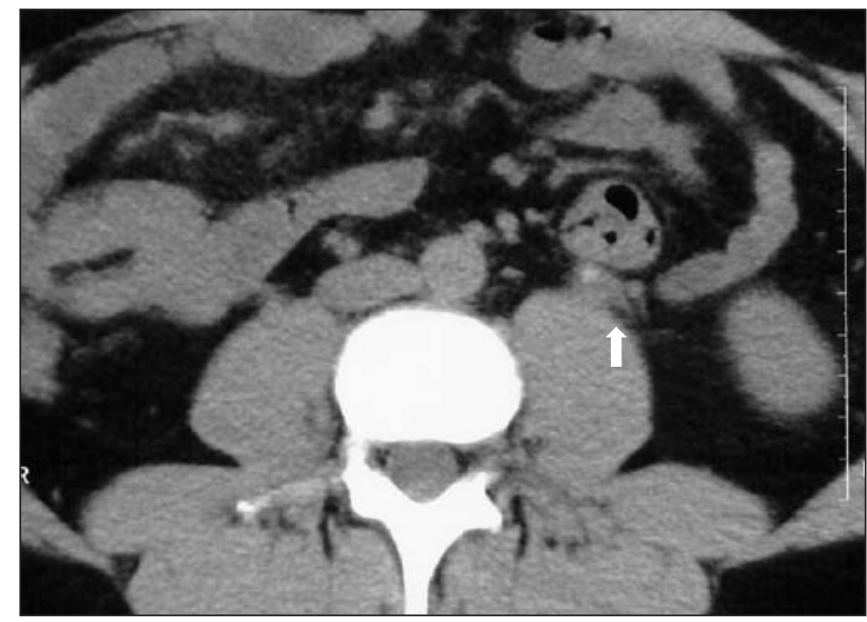

Figura 6. Sinal do halo. Tomografia computadorizada helicoidal sem contraste do abdome evidencia edema da parede ureteral que circunda o cálculo (setas). 


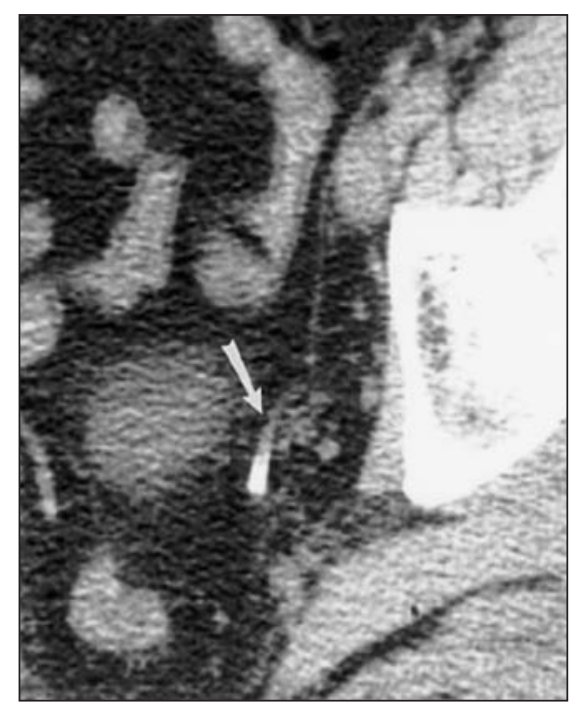

A

Figura 7. Sinal da cauda de cometa. Tomografia computadorizada helicoidal sem contraste do abdome demonstra calcificação vascular (flebólito) em cauda de cometa (setas).

rentes, aqueles de baixa atenuação ou pequenas dimensões, a eliminação recente e a não visualização do cálculo devido a artefatos respiratórios ou escassez de gordura retroperitoneal.

Além disso, os sinais secundários são de fundamental importância para a determinação do grau de obstrução urinária secundária à litíase, uma vez que apenas pacien-

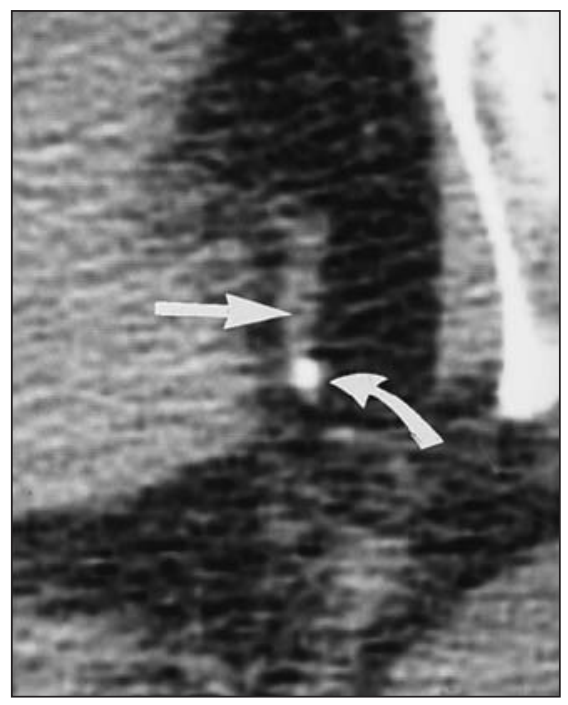

B

tes com obstrução desenvolvem nefromegalia, dilatação do sistema coletor/ureteral unilateral, heterogeneidade da gordura perirrenal, líquido perirrenal e perda da pirâmide renal hiperdensa.

\section{REFERÊNCIAS}

1. Smith RC, Rosenfield AT, Choe KA, et al. Acute flank pain: comparison of non-contrast-enhanced
CT and intravenous urography. Radiology 1995; 194:789-94.

2. Sheafor DH, Hertzberg BS, Freed KS, et al. Nonenhanced helical CT and US in the emergency evaluation of patients with renal colic: prospective comparison. Radiology 2000;217:792-7.

3. Smith RC, Verga M, Dalrymple N, McCarthy S, Rosenfield AT. Acute ureteral obstruction: value of secondary signs on helical unenhanced CT. AJR 1996;167:1109-13.

4. Dalrymple NC, Casford B, Raiken DP, Elsass KD, Pagan RA. Pearls and pitfalls in the diagnosis of ureterolithiasis with unenhanced helical CT. RadioGraphics 2000;20:439-47.

5. Guest AR, Cohan RH, Korobkin M, et al. Assessment of the clinical utility of the rim and comet-tail signs in differentiating ureteral stones from phleboliths. AJR 2001;177:1285-91.

6. Varanelli MJ, Coll DM, Levine JA, Rosenfield AT, Smith RC. Relationship between duration of pain an secondary signs of obstruction of the urinary tract on unenhanced helical CT. AJR 2001;177: $325-30$.

7. Roychowdhury A, Markis J, Colby JM, et al. Unilateral absence of the "white pyramid" sign on non contrast CT: a sign of tubular hydronephrosis? Presented at the 98th Meeting of the American Roentgen Ray Society, Scientific Session 33, San Francisco, California, April 26-May 1, 1998.

8. Katz DS, Hines J, Rausch DR, et al. Unenhanced helical CT for suspected renal colic. AJR 1999; 173:425-30.

9. Boridy IC, Nikolaidis P, Kawashima A, Goldman SM, Sandler CM. Ureterolithiasis: value of the tail sign in differentiating phleboliths from ureteral calculi at nonenhanced helical CT. Radiology 1999;211:619-21. 